\title{
EFFECT OF AUTONOMIC NERVOUS FUNCTION ON QT INTERVAL IN DOGS
}

\author{
Takuma HARADA, Junko ABE, Motohiro SHIOTANI, Yoshimasa HAMADA and Ikuo HORII \\ Worldwide Safety Sciences, Pfizer Global Research and Development, Nagoya Laboratories, Pfizer Japan Inc., \\ 5-2 Taketoyo, Aichi 470-2393, Japan
}

(Received November 22, 2004; Accepted April 26, 2005)

\begin{abstract}
The effects of drugs on the QT interval should be evaluated precisely in the early stages of drug development because QT prolongation can trigger the so-called torsades de pointes, a life-threatening polymorphic ventricular tachycardia. It has been reported that the QT interval is affected by autonomic nervous tone besides the heart rate. In this study, we investigated the direct effect of autonomic nervous tone on the QT interval using the parameters of heart rate variability in dogs, when the RR interval was constant $(400$ or $700 \mathrm{msec}$ ). Our results showed that the QT interval at the high HF (high vagal tone) or low LF/HF ratio (low sympathetic tone) was longer than that at the low HF (low vagal tone) or high LF/HF ratio (high sympathetic tone), when the RR intervals were constant, and that the effect of vagal tone on the QT interval might be somewhat stronger than that of the sympathetic tone. The present observations would support the idea that sympathetic as well as parasympathetic tone regulates QT interval and that QT interval may be controlled physiologically by myocardial autonomic nerves via and not via a sinus node. Therefore, a more precise correction formula of QT interval could be established using autonomic parameters other than RR interval (heart rate), while the QT interval is widely known to be dependent on the RR interval or heart rate.
\end{abstract}

KEY WORDS: Autonomic nervous tone, QT interval, Dog, Holter electrocardiogram

\section{INTRODUCTION}

QT interval prolongation can trigger the lifethreatening polymorphic ventricular tachycardia called torsades de pointes. Indeed, the development of some potentially useful drug candidates has been discontinued and some drugs have been withdrawn from the market in recent years because of the risk of torsades de pointes indicated by QT interval prolongation. Therefore, prolongation of the QT interval should be evaluated precisely in the early stages of drug development. The importance of the assessment of the potential of pharmaceuticals used in humans to induce QT interval prolongation has also been discussed in the ICH S7B/ E-14 guidelines.

As for the variations of the QT interval, some studies have reported that QT interval or corrected QT (QTc) interval exhibited a circadian rhythm in humans (Sarma et al., 1990; Smetana et al., 2003), but others reported a nonexistent or blunt circadian pattern of the
QTc interval (Morganroth et al., 1991; Molnar et al., 1996). This discrepancy may be partially due to differences in the heart rate correction formula used to calculate the QT interval, and in experimental conditions. It was also reported that the variation of QT interval might correlate with that of autonomic nervous function in a way independent from the heart rate when the QT interval is determined at a heart rate of 60 beats $/ \mathrm{min}$ in humans (Murakawa et al., 1992). However, the authors of that study did not estimate the effects of sympathetic and parasympathetic activity on the QT interval in a fully quantitative manner.

Heart rate variability (HRV) is a quantitative marker of autonomic activity. At least two main spectral components, low and high frequency (LF and HF) powers, are distinguished in a spectrum analyzed from short-term RR interval variation of the electrocardiogram (Task Force of the European Society of Cardiology and the North American Society of Pacing Electrophysiology, 1996). Sympathetic or vagal tone 
can be estimated from the LF and HF powers or their ratio. High frequency (HF) power reflects vagal activity and the low frequency (LF) to HF ratio (LF/HF) reflects sympathetic modulations, respectively. They are convenient indexes of parasympathetic and sympathetic interaction (Pagani et al., 1986).

In this study, we compared the QT interval at the same RR interval over time to clarify QT interval variation independent from heart rate, and also investigated the effect of autonomic nervous tone on the QT interval by measuring HRV parameters.

\section{MATERIALS AND METHODS}

\section{Animals}

Seven female beagle dogs (5.78-8.06 kg, 8-10 months old) were obtained from Marshall Farms USA (NY, USA) via Charles River Japan Inc. (Yokohama, Japan). The dogs were housed individually in metal cages in a climate-controlled room (temperature 21$25^{\circ} \mathrm{C}$; relative humidity $40-70 \%$ ) and a 12 -hr light (6:00-18:00)/ 12-hr dark cycle. They were given a certified pellet diet (250 g/day; DS-A, Oriental Yeast Co., Ltd., Tokyo, Japan) once daily, and municipal drinking water, further purified by reverse osmosis, was provided without limitations. All procedures involving animals were approved by the Animal Ethics Committee of Pfizer Global Research \& Development Nagoya Laboratories, and were performed in accordance with the NIH Guide for the Care and Use of Laboratory Animals.

\section{Holter ECG recording}

For acclimation prior to the attachment of a Holter recorder, the animals had to wear a Holter jacket (Star Medical, Inc., Tokyo, Japan), which held the Holter recorder, for 2 days before the recordings. Adhesive electrodes (Skintact FS-50, Leonhard Lang $\mathrm{GmbH}$, Innsbruck, Austria) were attached to the skin of the manubrium sterni in the region of the second thoracic vertebra (-) and xiphoid process $(+)$, and those for the supplemental lead were placed on the right ( $(-)$ and left $(+)$ sides of the chest. The ground electrode was placed on the right abdominal region. The electrodes were connected to a Holter recorder (SM-60, Fukuda-Denshi, Tokyo, Japan) that had been placed in the pocket of the Holter jacket.

Electrocardiograms were recorded for approximately 24 hrs beginning between 9:00 and 10:30, and access into the room was limited during the monitoring: food was supplied from 10:30 to 11:00 and the room cleaned at 16:00 and 8:00.

\section{ECG data process}

An ECG processor analyzing system (Softron ECG Processor, Softron, Tokyo, Japan) was used to analyze Holter ECG data. The computer program first detected $\mathrm{R}$ waves, and then calculated the RR and QT intervals in sequential order for all ECG waves. RR intervals before and after an artifact were excluded from the analysis. Power spectral analysis was conducted for the series of RR intervals, and QT- and preceding RR-interval pairs were extracted for QT analysis.

\section{Power spectral analysis}

Power spectral analysis was conducted referring to a method for dogs (Matsunaga et al., 2001) as follows: Using a series of RR intervals for $102.4 \mathrm{sec}$ as short-term recording, data sets of 512 points were resampled every $200 \mathrm{msec}$, and simultaneously subjected to Hanning window treatment to improve the spectral appearance (Harris, 1978). This data set was analyzed with fast Fourier transformation to obtain a power spectrum of heart rate variability (HRV). The 1-hr mean power spectrum was obtained by calculating and collecting the mean of each instantaneous power spectrum for a period of $102.4 \mathrm{sec}$, satisfying the recording period for the frequency domain analysis (Task Force of the European Society of Cardiology and the North American Society of Pacing Electrophysiology, 1996). For this 1-hr mean power spectrum, the high frequency (HF) power, which reflects vagal activity, and the low frequency (LF) power were calculated by integrating the power spectral density in defined frequency bands ( 0.04 to $0.1 \mathrm{~Hz}$ for LF power and 0.1 to $0.6 \mathrm{~Hz}$ for HF power). The threshold frequency between LF and HF components was determined on the basis of the results of spectral analysis conducted in conscious dogs (Frazier et al., 2001; Matsunaga et al., 2001). The LF to $\mathrm{HF}(\mathrm{LF} / \mathrm{HF})$ ratio, which is a convenient index of sympathetic and parasympathetic interaction that reflects sympathetic modulations (Pagani et al., 1986; Task Force of the European Society of Cardiology and the North American Society of Pacing Electrophysiology, 1996), was also calculated. Then we picked up the 1-hr period which showed the highest or lowest vagal activity, i.e. the time during which the highest or lowest mean HF power was recorded, respectively, to compare QT intervals at the same RR interval. The same procedure was conducted for the highest and lowest LF/HF ratio. 
Autonomic nervous tone and QT interval in dogs.

\section{Comparison of QT intervals at RR interval of 400 or $700 \mathrm{msec}$}

Using the QT- and preceding RR-interval pairs obtained from the ECG data, we collected all the pairs at RR interval of $400 \mathrm{msec}$ every $1 \mathrm{hr}$ in each animal. Then the QT intervals at RR interval of $400 \mathrm{msec}$ were compared between the 1-hr period showing the highest HF power and the 1-hr period showing the lowest HF power. The same procedure was repeated for $\mathrm{LF} / \mathrm{HF}$ ratios at an RR interval of 400 msec. For the long RR interval of $700 \mathrm{msec}$ as well as the short RR interval of $400 \mathrm{msec}$, HF power and LF/HF ratio were analyzed in a similar manner.

\section{Statistical analysis}

The mean QT intervals were compared by $t$-tests. Student's $t$-test was used if a preliminary $\mathrm{F}$ test did not show a significant variance homogeneity at the $\alpha=$ 0.05 level. If there was significant variance heterogeneity, Welch's $t$-test was used for the comparison (Yoshimura, 2000). Statistical significance was set at $\mathrm{p}<0.05$.

\section{RESULTS}

\section{Power spectral analysis}

The mean heart rate, $\mathrm{HF}, \mathrm{LF}$ and $\mathrm{LF} / \mathrm{HF}$ ratio for $24 \mathrm{hrs}$ in the $7 \mathrm{dogs}$ were 85 beats/min, $13962 \mathrm{msec}^{2}$, $2546 \mathrm{msec}^{2}$ and 0.17 , respectively (Table 1), and mean power spectral analysis for $24 \mathrm{hrs}$ showed a high HF power peak (Fig. 1A). The power spectra for 1-hr period showed various patterns. Fig. 1B shows an extremely high HF peak but Fig. 1C does not show any clear HF peak.

The mean HF for $1 \mathrm{hr}$ in each animal showed maximum value early in the morning at 4:00 - 6:00, and the mean $\mathrm{LF} / \mathrm{HF}$ value for $1 \mathrm{hr}$ became maximal when the animal room was cleaned (8:00 or 16:00).

Table 1. Mean values of power spectral analysis for $24 \mathrm{hrs}$ in $7 \mathrm{dogs}$.

\begin{tabular}{lc}
\hline Variable & Mean \pm SD \\
\hline Heart rate (beat/min) & $85 \pm 16$ \\
HF $\left(\mathrm{msec}^{2}\right)$ & $13962 \pm 4248$ \\
LF $\left(\mathrm{msec}^{2}\right)$ & $2546 \pm 2151$ \\
LF/HF & $0.17 \pm 0.10$ \\
HF $(\mathrm{nu})$ & $78.5 \pm 6.9$ \\
LF $(\mathrm{nu})$ & $12.9 \pm 5.5$ \\
\hline
\end{tabular}

\section{Comparison of QT intervals at constant RR inter-} vals

In the preliminary analysis, the QT interval at RR intervals of 400, 500, 600 or $700 \mathrm{msec}$ were compared between the 1-hr period that showed the highest HF power and the 1-hr period that showed the lowest HF power. The QT interval was longer at all RR intervals examined of 400 - $700 \mathrm{msec}$ when HF power was highest compared with that when HF power was lowest (Fig. 2. A and B). In addition, the QT interval tended to be even longer when the RR interval was prolonged from 400 to $700 \mathrm{msec}$ at the lowest or highest HF power (Fig. 2. A and B). Based on these results, the QT interval at 400 or $700 \mathrm{msec}$, covering a wide range of heart rate (86-150 beats/min), was selected to compare the 1-hr period that showed the highest HF power with the 1-hr period that showed lowest HF power in all dogs.

Fig. 3 shows representative ECG waveforms recorded at the highest (upper trace) and lowest (lower trace) HF powers at an RR interval of $400 \mathrm{msec}$. The QT interval at the highest HF power was obviously longer than that at the lowest HF power even if the RR interval was the same.

The QT intervals at the highest HF power were significantly longer than those at the lowest HF power in all dogs (Fig. 4A), and at the highest HF, the mean QT interval in the 7 dogs was $22 \mathrm{msec}$ longer than that at the lowest HF when the RR interval was $400 \mathrm{msec}$, and $16 \mathrm{msec}$ longer when the RR interval was 700 msec (Fig. 4B). Conversely, the QT intervals at the lowest $\mathrm{LF} / \mathrm{HF}$ ratio were longer than those at the highest LF/HF ratio in 5 of $7 \mathrm{dogs}$ (Fig. 5A), and at the lowest $\mathrm{LF} / \mathrm{HF}$ ratio, the mean QT interval in the $7 \mathrm{dogs}$ was $14 \mathrm{msec}$ longer than that at the highest LF/HF ratio when the RR interval was $400 \mathrm{msec}$ and $10 \mathrm{msec}$ longer when the RR interval was $700 \mathrm{msec}$ (Fig. 5B).

The QT interval became longer as the RR interval was prolonged from 400 to $700 \mathrm{msec}$ at the lowest and highest HF powers, and the lowest and highest LF/HF ratio in all the animals.

\section{DISCUSSION}

Holter, telemetry or restraint ECG recordings are generally used for pharmacological or toxicological studies in conscious dogs. Among these 3 methods, Holter recording presents the following advantages: i) it is not necessary to restrain the animals to record the ECG, avoiding thereby animal excitement; ii) it is easy to attach the electrodes to dogs and it is not necessary 
to acclimatize the dogs to the Holter jacket for a long period; iii) it is not necessary to implant any device, and thus there is no need for a recovery period; iv) it is possible to obtain a lot of ECG data during $24 \mathrm{hrs}$ to investigate the effect of treatment. Because of these advantages, we used Holter recording as the most convenient procedure to investigate the effect of treatment on ECG.

The mean values of heart rate, HF and LF pow- ers, and LF/HF ratio for $24 \mathrm{hrs}$ in the 7 dogs (Table 1) were comparable to those reported in other studies for dogs (Matsunaga et al., 2001; Takeuchi and Harada, 2002). The HF power was extremely high and the LF/ $\mathrm{HF}$ ratio was low in the dogs compared to those in other experimental animals (Hashimoto et al., 1999; Kuwahara et al., 1999; Akita et al., 2002). This difference may be due to the following reason. The high HF power is probably caused by RR interval variation due
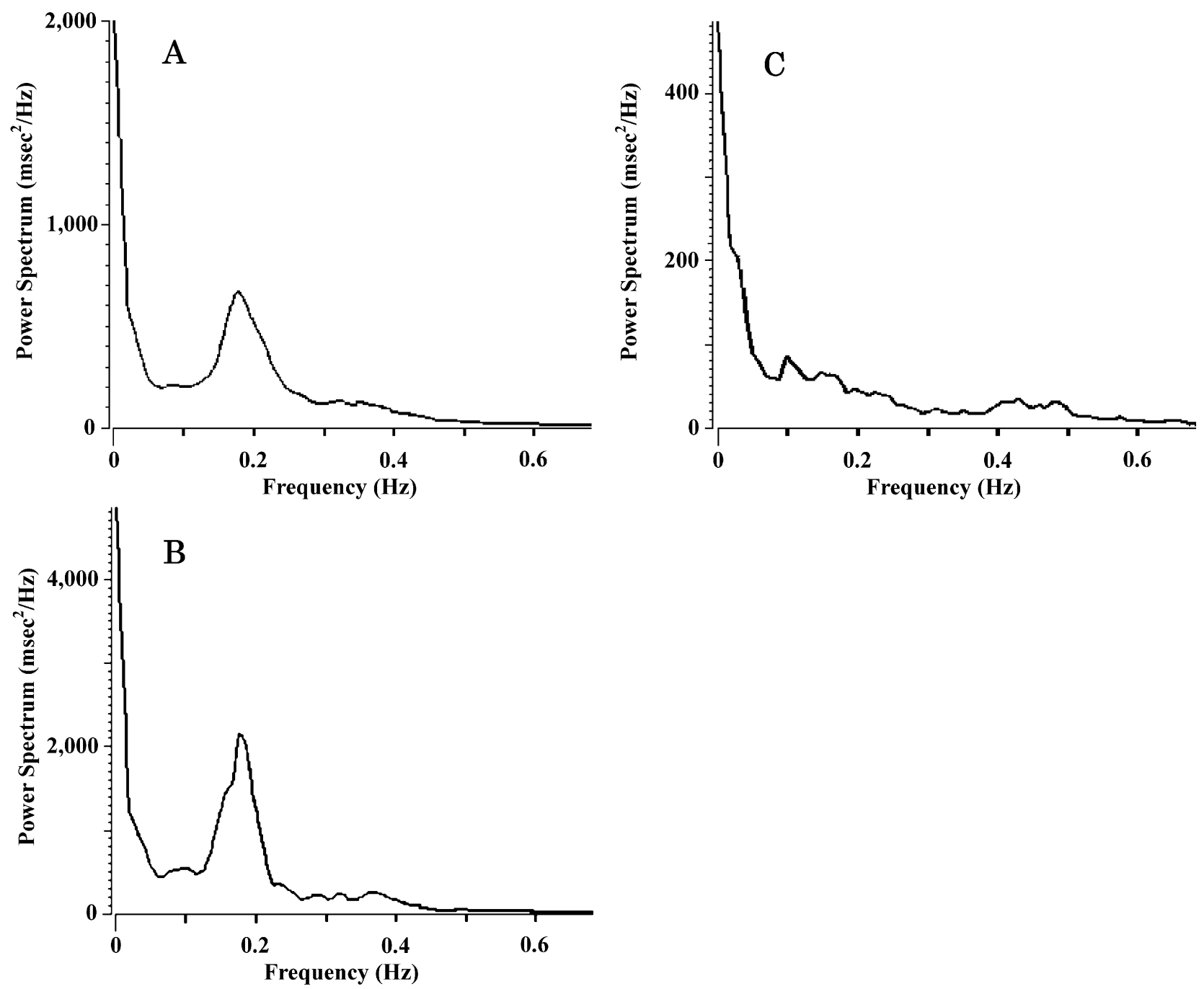

Fig. 1. Representative spectral power for heart rate in a beagle dog.

Power spectral analysis was conducted for the series of RR intervals from Holter ECG data. The 1-hr or 24-hr mean power spectra were obtained by calculating and collecting the mean of each instantaneous power spectrum for a period of 102.4 sec. A: Mean spectral power for $24 \mathrm{hrs}$

B: One-hour mean spectral power at the highest HF power

C: One-hour mean spectral power at the lowest HF power 
Autonomic nervous tone and QT interval in dogs.

to the respiratory sinus arrhythmia frequently observed in dogs and suggests a predominance of parasympathetic nervous activity.

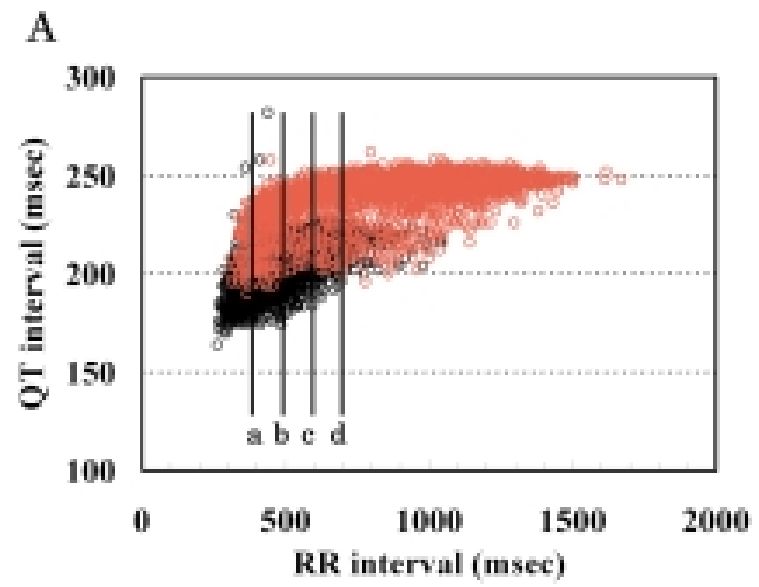

B

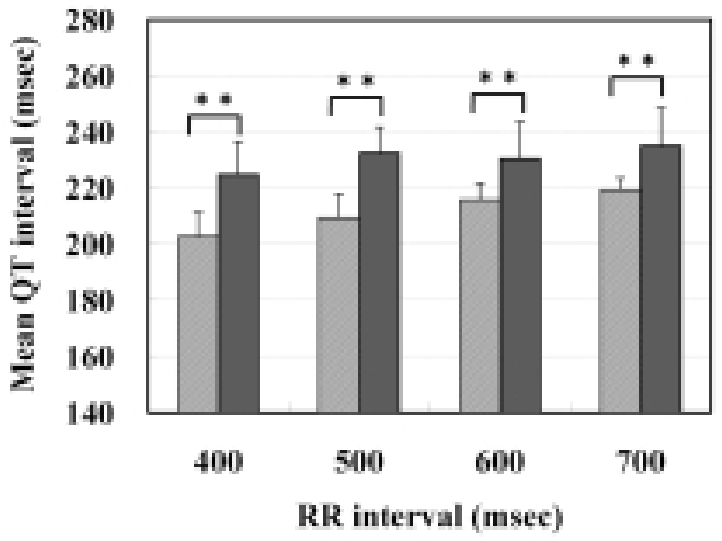

Fig. 2. QT-RR relationship at high and low HF powers in a beagle dog.

All the QT- and preceding RR-interval pairs for $1 \mathrm{hr}$ in a dog were extracted from Holter ECG data. The QT interval from each heart beat was plotted against the previous RR interval (A). QT-RR relationship (A) or QT interval (B) at high HF power was compared with that at low HF power in a beagle dog.

A: QT interval as a function of the RR interval at high (red circle) and low (black circle) HF powers in a beagle dog.

a: $400 \mathrm{msec}, \mathrm{b}: 500 \mathrm{msec}, \mathrm{c:} 600 \mathrm{msec}, \mathrm{d}: 700$ msec

B: Comparison of the mean QT interval between the highest and lowest HF powers at RR intervals of 400, 500, 600 and $700 \mathrm{msec}$. Data are shown as the mean $\pm \mathrm{SD}(\mathrm{n} \geq 21)$.

$\square$ : highest HF power, $\square$ : lowest HF power $* *: \mathrm{p}<0.01$
Comparing the QT intervals as a function of RR interval, the QT interval became longer as the RR interval was prolonged at the lowest and highest $\mathrm{HF}$ powers, and the lowest and highest $\mathrm{LF} / \mathrm{HF}$ ratios in all the animals (Figs. 4 and 5): the QT interval showed an inverse heart rate-dependency which is commonly seen in ECGs of humans and animals (Furushima et al., 1998; Hassimoto et al., 2002).

A number of researchers have established formulas to calculate the corrected QT interval based on heart rate or RR interval, such as Bazett's formula in humans and animals, because changes of the QT interval correlated inversely with changes in heart rate (Bazett, 1920; Matsunaga et al., 1997; Hassimoto et al., 2002). However, these QT correction formulas overcorrect the QT interval at a long RR interval and undercorrect it at a short RR interval, because these formulas are not suitable to correct the QT interval when the RR interval is very short or long (Smetana et al., 2003). Thus, the correction formula for QT interval does not fit well for a wide range of RR intervals. To exclude the unexpected effect of correction for QT interval and to quantitatively estimate the effect of autonomic activity on the QT interval, the same RR

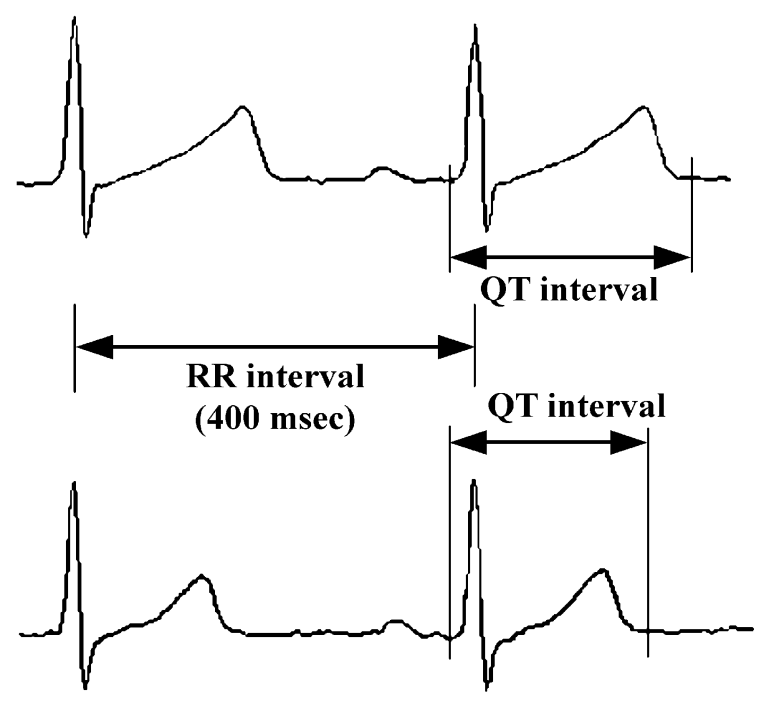

$\overline{100 \mathrm{msec}}$

Fig. 3. Representative ECG traces in a dog at an RR interval of $400 \mathrm{msec}$.

The QT interval of an ECG trace at the highest HF power (upper trace) was compared to that at the lowest HF power (lower trace) at an RR interval of 400 msec. 
A

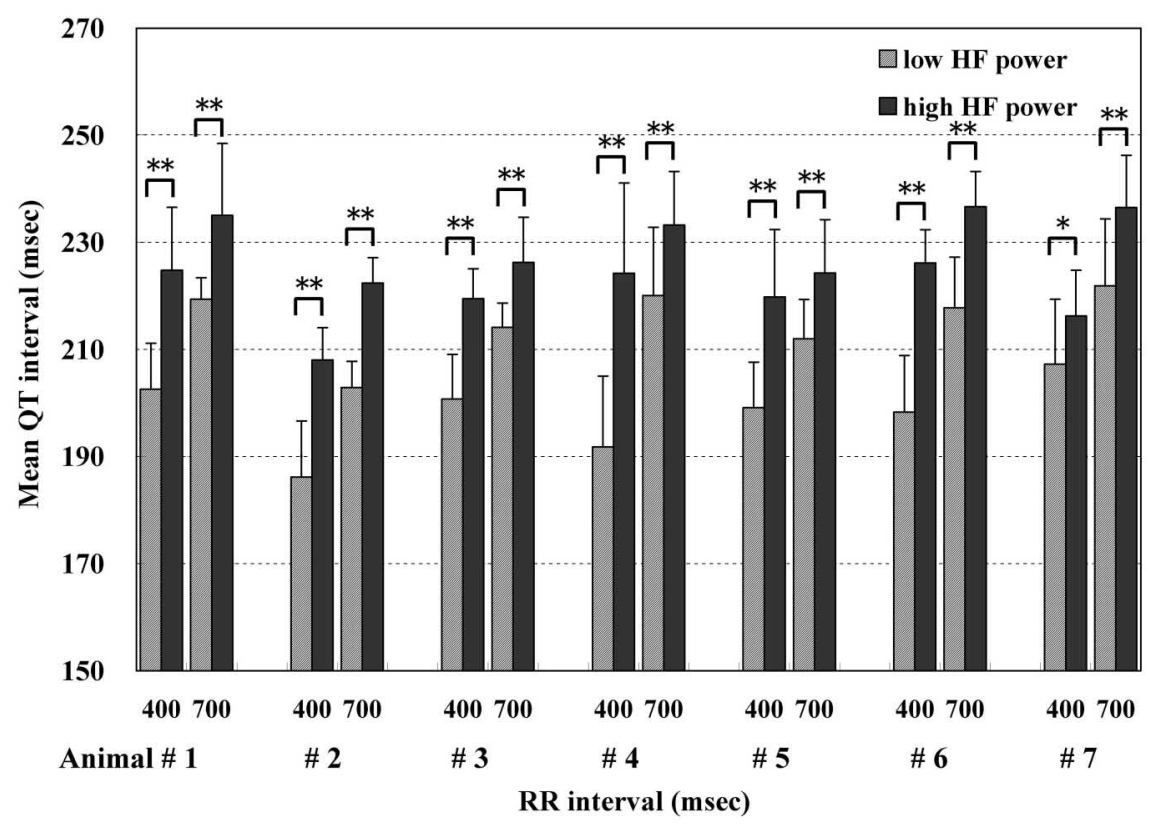

B

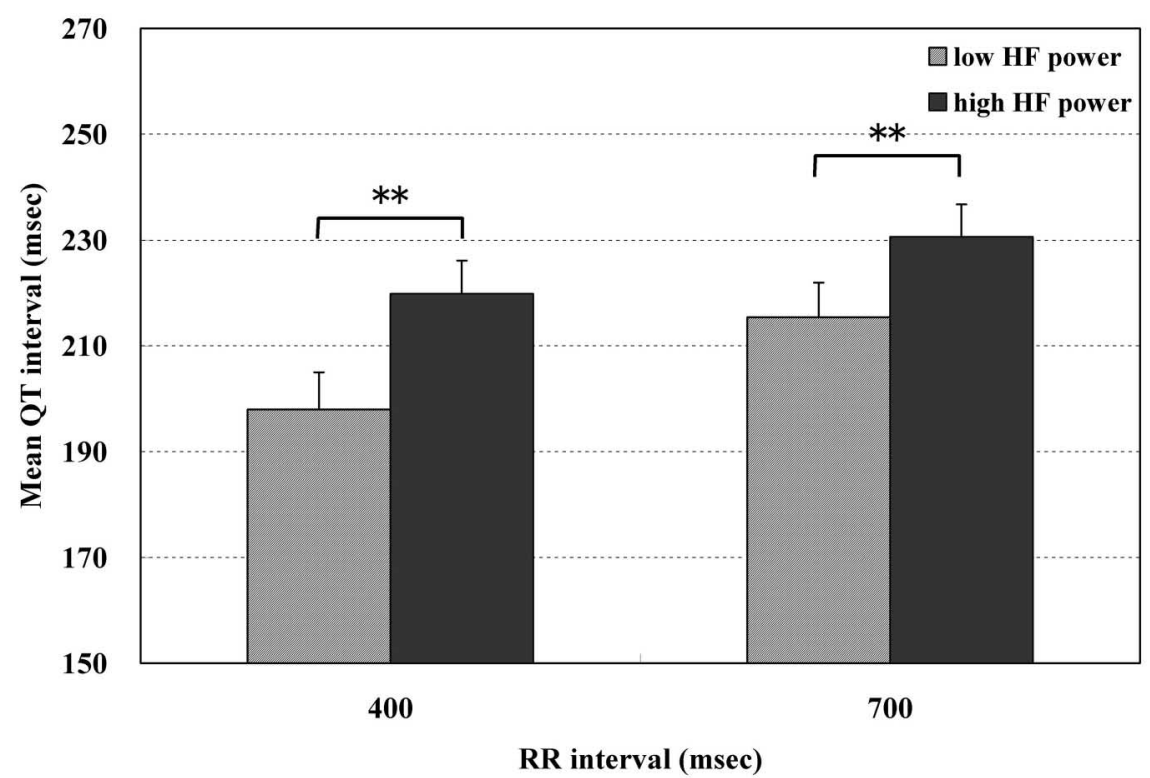

Fig. 4. Comparison of the mean QT interval between the lowest and highest HF powers at an RR interval of 400 or $700 \mathrm{msec}$ in the 7 dogs.

A: In each dog, the mean QT interval at RR interval of 400 or $700 \mathrm{msec}$ was compared between the 1-hour period showing the highest HF power and the 1-hour period showing the lowest HF power. Data are shown as the mean \pm SD ( $\mathrm{n} \geq 8$ ).

B: The difference in the mean QT interval in the 7 dogs was compared between the lowest and highest HF power at an RR interval of 400 or $700 \mathrm{msec}$. Data are shown as the mean $\pm \operatorname{SD}(n=7)$.

400: RR interval of $400 \mathrm{msec}$; 700: RR interval of $700 \mathrm{msec}$.

$* *: \mathrm{p}<0.01, *: \mathrm{p}<0.05$ 
Autonomic nervous tone and QT interval in dogs.

A

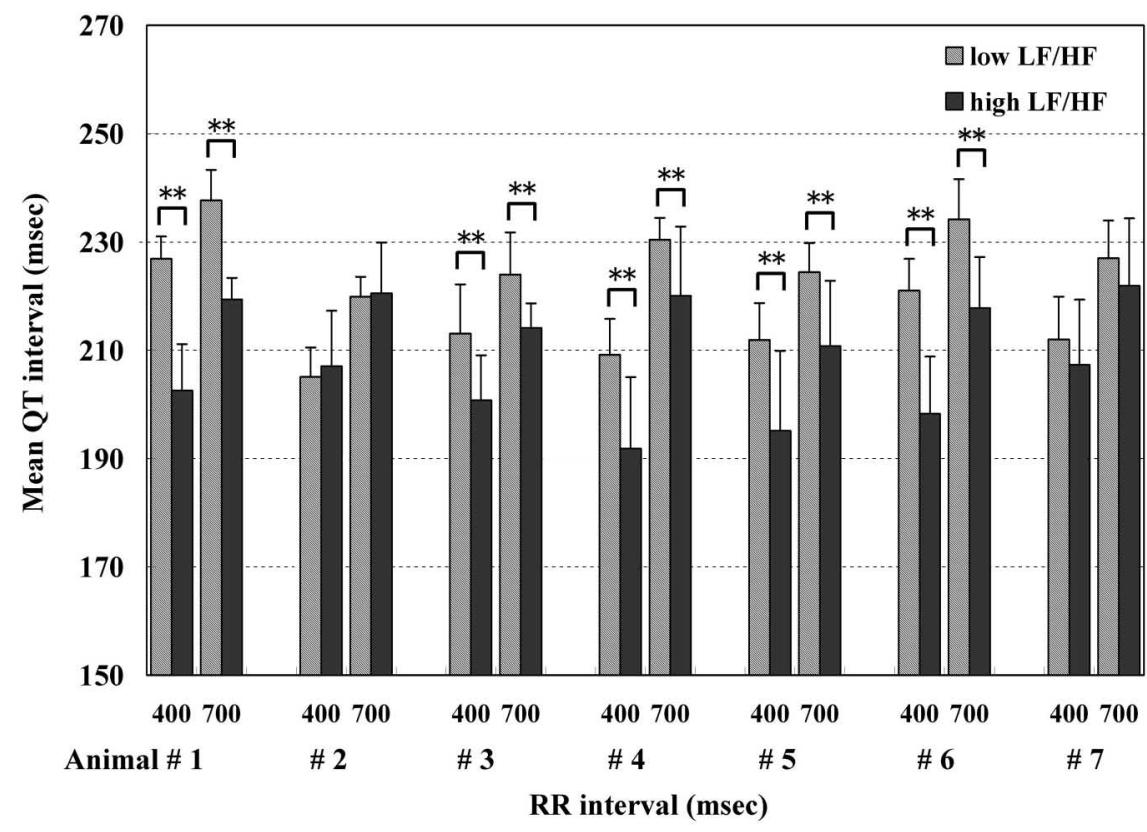

B

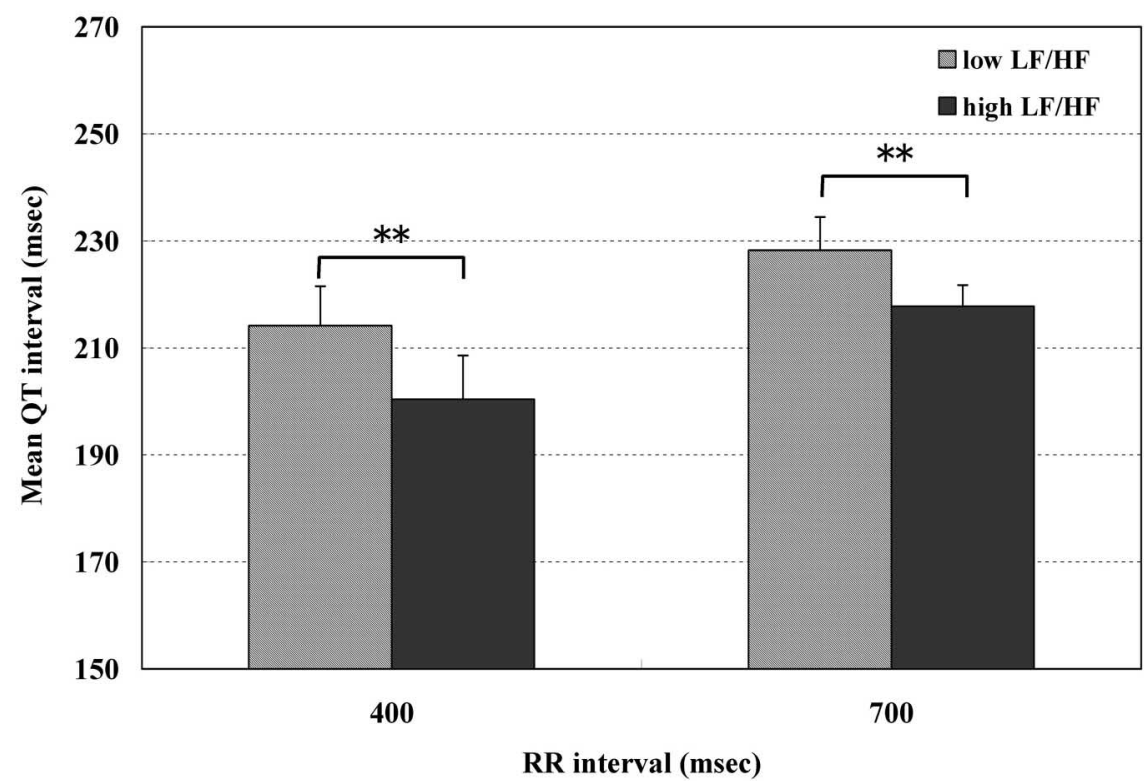

Fig. 5. Comparison of the mean QT interval between the lowest and highest LF/HF ratio at an RR interval of 400 or $700 \mathrm{msec}$ in the 7 dogs.

A: In each dog, the mean QT interval at RR interval of 400 or $700 \mathrm{msec}$ was compared between the 1-hour period showing the lowest LF/HF ratio and the 1-hour period showing the highest LF/HF ratio. Data are shown as the mean \pm SD $(n \geq 19)$.

B: The difference in the mean QT interval in the 7 dogs was compared between the lowest and highest LF/HF ratio at an RR interval of 400 or $700 \mathrm{msec}$. Data are shown as the mean $\pm \operatorname{SD}(n=7)$.

400: RR interval of $400 \mathrm{msec}$; 700: RR interval of $700 \mathrm{msec}$.

$* *: \mathrm{p}<0.01$ 
interval at 400 and $700 \mathrm{msec}$ was used to analyze the relation between QT interval and autonomic activity in this experiment. Some investigators showed that the physiologic relation between QT interval and heart rate (RR interval) was mainly under parasympathetic control by use of autonomic nervous blockade or an agonist (Browne et al., 1982; Lecocq et al., 1989), but others have speculated that both vagal and sympathetic tones were related to changes in the QT interval (Fananapazir et al., 1983; Kawataki et al., 1984). Our result showed that the QT interval at the highest HF power (highest vagal tone) or lowest LF/HF ratio (lowest sympathetic tone) was longer than that at the lowest HF power or highest LF/HF ratio, when the RR interval was constant (Figs. 4 and 5). It was also suggested that the effect of vagal tone on the QT interval might be somewhat stronger than that of sympathetic tone because HF power, which reflects vagal tone, is very high in dogs compared to that in other animals (Table 1) and no significant differences in the QT interval between the lowest and highest $\mathrm{LF} / \mathrm{HF}$ ratios were detected in 2 animals (Fig. 5). QT interval represents the time required for complete ventricular depolarization and repolarization. These changed with the vagal and sympathetic nervous tone independent from the heart rate (Figs. 4 and 5). The present observations would support the idea that sympathetic as well as parasympathetic tone regulates QT interval and that

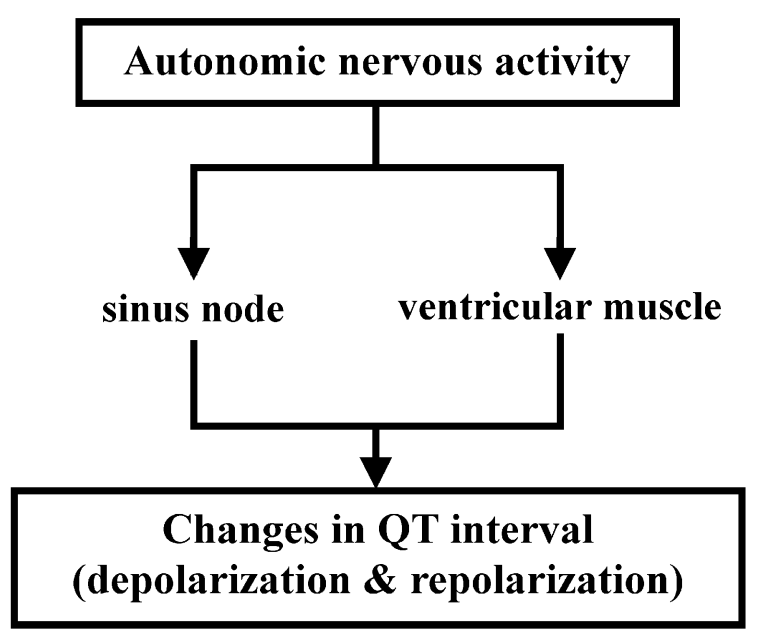

Fig. 6. Hypothetic scheme from the result of the present study regarding the effect of autonomic activity on QT interval in ventricular myocardium. Autonomic nerves may regulate the QT interval in cardiac ventricular muscle via and not via a sinus node.
QT interval may be controlled physiologically by myocardial autonomic nerves via and not via a sinus node (Fig. 6). Therefore, a more precise correction formula of QT interval could be established using autonomic parameters other than RR interval (heart rate), while the QT interval is widely known to be dependent on the RR interval or heart rate (Bazett, 1920; Matsunaga et al., 1997; Hassimoto et al., 2002).

There are some reports on the fluctuation of QT interval in humans (Browne et al., 1983; Murakawa et al., 1992), but the detailed mechanism remains unclear. Therefore, in pharmacological or toxicological studies, the effect of drugs on the QT or QTc interval must be evaluated considering the fluctuation of the interval. From the result of this study on autonomic balance, the mechanism of QT interval fluctuations might be partially due to temporal changes in autonomic nervous tone. In fact, heart rate, which may be regulated by autonomic tone, shows fluctuations due to changes in sympathetic and parasympathetic balance in rats, guinea pigs, dogs, miniature swine and humans (Hashimoto et al., 1999; Kuwahara et al., 1999; Matsunaga et al., 2001; Akita et al., 2002; Smetana et al., 2003).

In this study, we demonstrate that changes in autonomic function would directly alter the ventricular depolarization and repolarization time, i.e. QT interval. However, well controlled or time-matched experiments are needed to further elucidate the detailed autonomic nervous function involved in the regulation of the QT interval.

\section{ACKNOWLEDGEMENT}

We thank Mr. Atsushi Takai for his excellent care of the study animals.

\section{REFERENCES}

Akita, M., Ishii, K., Kuwahara, M. and Tsubone, H. (2002): Power spectral analysis of heart rate variability for assessment of diurnal variation of autonomic nervous activity in guinea pigs. Exp. Anim., 51, 1-7.

Bazett, H.C. (1920): An analysis of the time relations of electrocardiograms. Heart, 7, 353-370.

Browne, K.F., Prystowsky, E., Heger, J.J., Chilson, D.A. and Zipes, D.P. (1983): Prolongation of the Q-T interval in man during sleep. Am. J. Cardiol., 52, 55-59.

Browne, K.F., Zipes, D.P., Heger, J.J. and Prystowsky, 
Autonomic nervous tone and QT interval in dogs.

E.N. (1982): Influence of the autonomic nervous system on the Q-T interval in man. Am. J. Cardiol., 50, 1099-1103.

Fananapazir, L., Bennett, D.H. and Faragher, E.B. (1983): Contribution of heart rate to QT interval shortening during exercise. Eur. Heart J., 4, 265271.

Frazier, S.K., Moser, D.K. and Stone, K.S. (2001): Heart rate variability and hemodynamic alterations in canines with normal cardiac function during exposure to pressure support, continuous positive airway pressure, and a combination of pressure support and continuous positive airway pressure. Biol. Res. Nurs., 2, 167-174.

Furushima, H., Niwano, S., Chinushi, M., Ohhira, K., Abe, A. and Aizawa, Y. (1998): Relation between bradycardia dependent long QT syndrome and QT prolongation by disopyramide in humans. Heart, 79, 56-58.

Harris, F.J. (1978): On the use of windows for harmonic analysis with the discrete Fourier transform. IEEE Proc., 66, 51-83.

Hashimoto, M., Kuwahara, M., Tsubone, H. and Sugano, S. (1999): Diurnal variation of autonomic nervous activity in the rat: Investigation by power spectral analysis of heart rate variability. J. Electrocardiol., 32, 167-171.

Hassimoto, M., Harada, T., Kaga, N., Murano, H. and Obata, M. (2002): Accurate evaluation of QT interval in conscious rhesus monkeys (Macaca mulatta) by use of Holter ECG. J. Electrocardiol., 35, 333-342.

Kawataki, M., Kashima, T., Toda, H. and Tanaka, H. (1984): Relation between QT interval and heart rate. applications and limitations of Bazett's formula. J. Electrocardiol., 17, 371-375.

Kuwahara, M., Suzuki, A., Tsutsumi, H., Tanigawa, M., Tsubone, H. and Sugano, S. (1999): Power spectral analysis of heart rate variability for assessment of diurnal variation of autonomic nervous activity in miniature swine. Lab. Anim. Sci., 49, 202-208.

Lecocq, B., Lecocq, V. and Jaillon, P. (1989): Physiologic relation between cardiac cycle and QT duration in healthy volunteers. Am. J. Cardiol., 64, 481-486.

Matsunaga, T., Harada, T., Mitsui, T., Inokuma, M., Hashimoto, M., Miyauchi, M., Murano, H. and Shibutani, Y. (2001): Spectral analysis of circadian rhythms in heart rate variability of dogs. Am. J. Vet. Res., 62, 37-42.
Matsunaga, T., Mitsui, T., Harada, T., Inokuma, M., Murano, H. and Shibutani, Y. (1997): QT corrected for heart rate and relation between QT and RR intervals in beagle dogs. J. Pharmacol. Toxicol. Methods., 38, 201-209.

Molnar, J., Zhang, F., Weiss, J., Ehlert, F.A. and Rosenthal, J.E. (1996): Diurnal pattern of QTc interval: How long is prolonged? Possible relation to circadian triggers of cardiovascular events. J. Am. Coll. Cardiol., 2, 76-83.

Morganroth, J., Brozovich, F.V., McDonald, J.T. and Jacobs, R.A. (1991): Variability of the QT measurement in healthy men, with implications for selection of an abnormal QT value to predict drug toxicity and proarrhythmia. Am. J. Cardiol., 67, 774-776.

Murakawa, Y., Inoue, H., Nozaki, A. and Sugimoto, T. (1992): Role of sympathovagal interaction in diurnal variation of QT interval. Am. J. Cardiol., 69, 339-343.

Pagani, M., Lombardi, F., Guzzetti, S., Rimoldi, O., Furlan, R., Pizzinelli, P., Sandrone, G., Malfatto, G., Dell'Orto, S., Piccaluga, E., Turiel, M., Baselli, G., Cerutti, S. and Malliani, A. (1986): Power spectral analysis of heart rate and arterial pressure variabilities as a marker of sympathovagal interaction in man and conscious dog. Circ Res., 59, 178-193.

Sarma, J.S., Venkataraman, K., Nicod, P., Polikar, R., Smith, J., Schoenbaum, M.P. and Singh, B.N. (1990): Circadian rhythmicity of rate-normalized QT interval in hypothyroidism and its significance for development of class III antiarrhythmic agents. Am. J. Cardiol., 66, 959-963.

Smetana, P., Batchvarov, V., Hnatkova, K., Camm, A.J. and Malik, M. (2003): Circadian rhythm of the corrected QT interval: Impact of different heart rate correction models. Pacing Clin. Electrophysiol., 26, 383-386.

Takeuchi, T. and Harada, E. (2002): Age-related changes in sleep-wake rhythm in dog. Behav. Brain Res., 136, 193-199.

Task Force of the European Society of Cardiology and the North American Society of Pacing Electrophysiology (1996): Heart Rate Variability: Standards of Measurement, Physiological Interpretation, and Clinical Use. Circulation, 93, 1043-1065.

Yoshimura, I. (2000): In "Statistical Analysis of Toxicological Data", pp. 26-42, Scientist, Tokyo (in Japanese). 